\title{
Aspectos atuais na fisiopatologia do edema macular diabético
}

\section{Recent aspects on physiopathology of diabetic macular edema}

Mário Martins dos Santos Motta'; Jacqueline Coblentz²; Laura Gomes Nunes de Melo

\section{RESUMO}

O edema macular é a principal causa de baixa visual em pacientes diabéticos. Seu mecanismo de formação é complexo e envolve alterações bioquímicas e estruturais. Os autores fazem uma revisão e atualização dos conceitos fisiopatológicos envolvidos na maculopatia diabética.

Descritores: Retinopatia diabetica/fisiopatologia; Edema macular; Diabetes Mellitus tipo 1/complicações; Diabetes Mellitus tipo 2/complicações

'Livre Docente, Professor adjunto e da Universidade Federal do Estado do Rio de Janeiro - UNIRIO - Rio de Janeiro (RJ), Brasil; Professor Titular da Faculdade de Medicina de Teresópolis - UNIFESO - Teresópolis (RJ), Brasil.

${ }^{2}$ Médica oftalmologista da Universidade Federal do Estado do Rio de Janeiro - UNIRIO - Rio de Janeiro (RJ), Brasil.

${ }^{3}$ Residente do Hospital dos Servidores do Rio de Janeiro - HSE - Rio de Janeiro (RJ), Brasil. 


\section{INTRODUÇÃO}

A retinopatia diabética é a segunda maior cau sa de cegueira legal nos países ocidentais, correspondendo a cerca de $19 \%$ dos casos de cegueira ${ }^{1-2}$. A maculopatia diabética, cuja principal manifestação é o edema macular, é a causa mais freqüente de perda significativa da função visual em diabéticos, com prevalência de 18 a $20 \%$, tanto em pacientes com diabetes tipo 1 quanto em diabetes tipo $2^{3}$. A perda visual pelo diabetes pode ser evitada ou minimizada, com controle clínico adequado e tratamentos locais e/ou sistêmicos. O conhecimento cada vez mais detalhado da fisiopatologia da retinopatia diabética tem permitido chegar a métodos de prevenção e tratamento mais eficazes.

É importante ressaltar que grande parte dos estudos laboratoriais relacionados à gênese da retinopatia diabética corresponde à experimentação em modelos animais. Devem, portanto, ser interpretados com a devida cautela em relação ao que ocorre em humanos.

\section{Alterações bioquímicas}

O fator mais importante para ocorrência e progressão da retinopatia diabética (RD) é a hiperglicemia crônica $^{4-8}$. Os mecanismos bioquímicos relacionados à hiperglicemia prolongada são: alteração na expressividade de genes; ligação não-enzimática de açúcares a proteínas; aumento no estresse oxidativo com excesso de produtos finais da oxidação e infiltração de células inflamatórias e produção de citocinas na retina de diabéticos ${ }^{5,8}$.

Via do sorbitol - são reações com as enzimas aldose redutase e sorbitol desidrogenase, cujas expressões genéticas são reguladas pela hiperhexosemia crônica. A aldose redutase usa a forma reduzida do nucleotídeo trifosforopiridina (NADPH) como cofator e reduz açúcares aldose a seus respectivos açúcares alcoólicos, que são oxidados para os ceto-açúcares pela sorbitol desidrogenase (exceto o galactiol); por exemplo a glicose, em níveis elevados, é reduzida a sorbitol pois as vias normais de metabolização estão saturadas. Esta última reação é lenta em algumas células, como o epitélio do cristalino e endotélio vascular, causando acúmulo do sorbitol em níveis tóxicos com espessamento da membrana basal dos capilares e formação precoce de catarata, observados em diabéticos ${ }^{8}$.
Glicolisação não-enzimática - As moléculas de glicose ligam-se ao grupamento epsilon-amino dos resíduos de lisina nas proteínas, e estas se entrecruzam (reação de Amadori) formando os produtos finais avançados da glicolisação (proteínas AGE) ${ }^{8}$. A mudança na estrutura das proteínas atuaria nas células e na matriz extracelular, favorecendo a instalação das complicações crônicas do diabetes ${ }^{5,8}$.

Diaglicerol, PCK e VEGF - a hiperglicemia prolongada causa acúmulo de diaglicerol nas células da retina e aorta de cães diabéticos, ativando a PCKâ. Níveis elevados do diaglicerol aumentam a atividade da PCK, cuja isoforma â parece mediar as ações do fator vascular de crescimento endotelial (VEGF) após a ligação deste com seus receptores de membrana $^{9-10}$. O VEGF manifesta-se sob quatro diferentes isoformas com 121 a 206 aminoácidos ${ }^{5}$. $\mathrm{Na}$ retina, o VEGF fosforila as proteínas das junções apertadas das células endoteliais vasculares, levando ao aumento da permeabilidade vascular ${ }^{11}$. O provável mecanismo de lesão na barreira hematoretiniana interna envolve alterações na estrutura das proteínas responsáveis pelas junções apertadas intercelulares (ocludina e ZO-1). Além disso, o VEGF também atua como principal fator angiogênico da retina, em reposta à hipóxia ${ }^{5,12-14}$.

As células retinianas sintetizam fatores fibroblásticos do crescimento ácido e básico (aFGF; bFGF). O bFGF estaria implicado nas formas proliferativas, podendo atuar de modo sinérgico com o VEGF na proliferação celular e angiogênese $e^{5,11}$.

O fator derivado do epitélio pigmentado (PEDF) é uma glicoproteína que regula o crescimento vascular na retina e promove diferenciação neural. Secretado pelas células ganglionares e pelas do epitélio pigmentado, inibe a angiogênese em modelos animais e culturas de células ${ }^{11}$. Hiperglicemia e hipóxia reduzem a produção de PEDF e estimulam o VEGF, o que sugere mecanismo regulatório, ${ }^{5,11}$. A concentração vítrea aumentada de PEDF correlacionase com redução da espessura da retina, em casos de edema retiniano ${ }^{11}$.

O aumento dos níveis de fator de crescimento da insulina1 (IGF-1), um polipeptídeo semelhante à cadeia A da insulina produzido no fígado sob influência do hormônio do crescimento $(\mathrm{GH})$, facilita a mitogênese e pode estar relacionado ao aparecimento de RD proliferativa em diabéticos tipo $1^{8}$.

Argumentos favoráveis à influência do GH e 
do IGF-1 nas formas proliferativas da RD incluem o fato de que a doença não ocorre antes da puberda$\mathrm{de}^{8,15}$, a hipofisectomia cirúrgica ou por irradiação foi utilizada com sucesso em casos que não responderam à fotocoagulação ${ }^{16}$, os níveis séricos e vítreos de IGF1 encontram-se elevados em diabéticos tipo1 com retinopatia proliferativa ${ }^{17}$ e alguns pacientes com diabetes tipo1 têm piora da RD após controle glicêmico rápido, acompanhado de elevação dos níveis séricos de IGF-1 $1^{18}$.

Além disso, é conhecido o fato de que a RD piora durante a gravidez, época em que existe aumento da liberação de $\mathrm{GH}$.

Estudos em camundongos recém-nascidos sugerem que o GH e o IGF-1 seriam fatores permissivos para o desenvolvimento de neovasos na retina, mas não sua causa direta ${ }^{19}$. Existem, ainda, características inflamatórias na RD, uma vez que se observou maior ativação leucocitária na retina de ratos diabéticos. Como resultado, há liberação de citocinas inflamatórias e moléculas de adesão, que aumentam a adesão dos leucócitos às paredes dos capilares retinianos. Esse mecanismo leva à estase capilar e oclusão, resultando em hipóxia.

\section{Alterações estruturais}

Histologicamente, as alterações mais precoces são espessamento da membrana basal capilar e redução no número de pericitos (células mesoteliais que envolvem as células do endotélio capilar e as vênulas). Fisiologicamente, estas estruturas inibem a vasoproliferação retiniana ${ }^{8}$.

A membrana basal é constituída por colágeno IV, laminina, entactina e heparan sulfato. Funciona como suporte para os vasos, inibe a proliferação endotelial e atua na permeabilidade seletiva ${ }^{5}$. Resíduos de heparan sulfato ligam-se aos fatores de crescimento aFGF e bFGF, reduzindo o potencial de ligação dos receptores de superfície celular. O espessamento da membrana basal afasta os pericitos do endotélio vascular e reduz os locais de ligação heparan sulfato-FGF, diminuindo a capacidade de inibir a proliferação das células vasculares ${ }^{8}$. A via do sorbitol está implicada nas alterações da membrana basal dos diabéticos, que além de encontrar-se espessada mostra vacuolização e deposição de colágeno fibrilar nos exames com microscopia eletrônica. A redução na produção de heparan sulfato, que tem carga elétrica negativa, e aumento de colágeno na mem- brana basal poderiam afetar a permeabilidade da membrana para diferentes íons, influindo na função da barreira hemato-retiniana ${ }^{5,8}$.

A redução do número de pericitos na RD favorece a dilatação dos capilares onde aqueles estão ausentes, sendo este o provável mecanismo de formação dos microaneurismas ${ }^{3,20}$. Os eventos que favorecem a apoptose seletiva dos pericitos ainda não foram esclarecidos 5 .

Os microaneurismas, as primeiras lesões clinicamente observadas na $\mathrm{RD}$, são pequenos pontos vermelhos à oftalmoscopia e hiperfluorescentes na fluoresceinografia ${ }^{520}$. Seu diâmetro varia entre $15-60 \mu \mathrm{m}$ e são mais numerosos no pólo posterior. Surgem inicialmente no leito venoso dos capilares e posteriormente nas arteríolas pré-capilares. Na RD avançada, há perda das células endoteliais com formação de capilares acelulares não-perfundidos ${ }^{8,20}$.

As dilatações capilares são permeáveis ao plasma com lipídios e proteínas, e seu extravasamento causa edema na retina. $\mathrm{O}$ acúmulo de fibrina nos aneurismas, ocluindo os vasos, agrava a hipóxia. Como resposta ao aumento de sorbitol nas células endoteliais, há dilatação venosa nas áreas adjacentes aos capilares nãoperfundidos ${ }^{21}$.

Quando a parede do microaneurisma se enfraquece, este pode se romper e causar a hemorragia retiniana. Na retina mais profunda, com células e axônios orientados verticalmente, a hemorragia será puntiforme ou em manchas; na camada mais superficial, com fibras nervosas orientadas horizontalmente, assume aspecto de "chama de vela" ${ }^{20}$.

A hipóxia progressiva causa isquemia na camada de fibras nervosas, resultando em bloqueio do fluxo axoplásmico e edema destas fibras (manchas algodonosas) $)^{3,20}$.

Outros sinais de hipóxia são dilatações segmentares e irregulares dos capilares, chamados de anormalidades microvasculares intra-retinianas (IRMA) e alterações das vênulas: beading e looping. O beading tem aspecto em rosário com dilatações e constrições intercaladas. O looping é uma área de desvio da vênula, em forma de laço. Seriam tentativas de formar circulação colateral ${ }^{5,20}$.

A progressão da isquemia leva ao surgimento de neovasos que inicialmente são finos, delicados e de baixa resistência. Em seguida há proliferação fibrosa, para sustentá-los. Nas etapas mais avançadas ocorre regressão do sistema vascular, contratura do tecido 
conjuntivo, espessamento do vítreo e tração sobre os neovasos e/ou retina, causando hemorragia vítrea, descolamento de retina, retinosquise e aumento das alterações fibróticas ${ }^{5,8}$.

Na RD, o rompimento da barreira hematoretiniana interna permite o extravasamento de líquido e constituintes plasmáticos (principalmente lipoproteínas) para o espaço intersticial da retina. $\mathrm{O}$ edema pode ser focal ou difuso e manifesta-se clinicamente como retina espessa e opacificada ${ }^{20}$.

No edema focal, há áreas de vazamento a partir de lesões capilares específicas, que pode estar associado aos exsudatos duros (precipitação de resíduos lipídicos, após a reabsorção do fluído derivado do extravasamento de lipoproteínas plasmáticas). Podem apresentar aspecto "circinado", e tendem a atingir a fóvea ${ }^{6}$.

O edema difuso é causado por dilatação capilar e vazamento devido a quebras extensas na barreira hemato-retiniana, sendo que os exsudatos duros são menos comuns. Define-se edema macular difuso pela presença de espessamento retiniano, visível à biomicroscopia, com superfície de dois diâmetros papilares ou maior, que acometa a zona avascular foveal $^{21}$.

Caso o extravasamento de líquido fique septado, constitui o edema macular cistóide, que coexiste mais com as formas difusas do que com as focais ${ }^{6}$.

\section{Conclusão}

A perda visual pela retinopatia diabética pode ser evitada ou minimizada. Para tanto, o conhecimento cada vez mais detalhado da fisiopatologia da retinopatia diabética deve ser buscado, para que seja possível chegar a novos métodos diagnósticos, e principalmente, novas opções terapêuticas.

\section{Abstract}

Macular edema is the leading cause of poor vision in diabetic patients. The mechanism of edema formation is complex and involves biochemical and structural changes. The authors review and update the physiopathologic concepts related to diabetic maculopathy.

Keywords: Diabetic retinopathy/physiopathology; Macular edema; Diabetes Mellitus, type 1/complications; Diabetes Mellitus, type 2/complications

\section{RefERÊNCIAS}

1. Klein R, Klein BE, Moss SE, Davis MD, DeMets DL. The Wisconsin epidemiologic study of diabetic retinopathy. III. Prevalence and risk of diabetic retinopathy when age at diagnosis is 30 or more years. Arch Ophthalmol. 1984; 102(4): 527-32.

2. Kempen JH, O'Colmain BJ, Leske MC, Haffner SM, Klein R, Moss SE, Taylor HR, Hamman RF; Eye Diseases Prevalence Research Group. The prevalence of diabetic retinopathy among adults in the United States. Arch Ophthalmol. 2004; 122(4):552-63.

3. Chew EY, Ferris III FL. Nonproliferative diabetic retinopathy. In: Ryan SJ, editor. Retina. 5th ed. St. Louis: Mosby; 2006. vol. II, cap. 67, seção 5. P. 1271-84.

4. Ministério da Saúde. Indicadores de morbidade e fatores de risco: D.10 Taxa de prevalência de diabete melito[Internet] [citado 2007 Dez 12]. Disponível em: http://tabnet.datasus. gov.br/cgi/idb2006/d10.htm

5. The effect of intensive treatment of diabetes on the development and progression of long-term complications in insulin-dependent diabetes mellitus. The Diabetes Control and Complications Trial Research Group. N Engl J Med. 1993; 329(14):977-86.

6. Intensive blood-glucose control with sulphonylureas or insulin compared with conventional treatment and risk of complications in patients with type 2 diabetes (UKPDS 33) UK Prospective Diabetes Study (UKPDS) Group. Lancet. 1998; 352(9131): 837-53. Erratum in: Lancet 1999; 354(9178):602

7. Retinopathy and nephropathy in patients with type 1 diabetes four years after a trial of intensive therapy. The Diabetes Control and Complications Trial/Epidemiology of Diabetes Interventions and Complications Research Group N Engl J Med. 2000; 342(6):381-9. Erratum in: N Engl J Med. 2000; 342(18):1376.

8. Augustin AJ, Dick HB, Koch F, Schmidt-Erfurth U. Correlation of blood-glucose control with oxidative metabolites in plasma and vitreous body of diabetic patients. Eur J Ophthalmol. 2002; 12(2): 94-101.

9. Aiello LP, Bursell SE, Clermont A, Duh E, Ishii H, Takagi C, et al. Vascular endothelial growth factor-induced retinal permeability is mediated by protein kinase $\mathrm{C}$ in vivo and suppressed by an orally effective beta-isoform-selective inhibitor. Diabetes. 1997; 46(9): 1473-80.

10. PCK-DRS2 Group, Aiello LP, Davis MD, Girach A, Kles KA, Milton RC, Sheetz MJ, et al. Effect of ruboxistaurin on visual loss in patients with diabetic retinopathy. Ophthalmology. 2006; 113(12): 2221-30.

11. Aiello LP, Avery RL, Arrigg PG, Keyt BA, Jampel HD, Shah ST, et al. Vascular endothelial growth factor in ocular fluid of patients with diabetic retinopathy and other retinal disorders. N Engl J Med. 1994; 331(22):1480-7. Comment in: N Engl J Med. 1994; 331(22):1519-20.

12. Avery RL, Pearlman J, Pieramici DJ, Rabena MD, Castellarin AA, Nasir MA, et al. Intravitreal bevacizumab (Avastin) in the treatment of proliferative diabetic retinopathy. Ophthalmology. 2006; 113(10):1695.e1-15.

13. Cunningham ET Jr, Adamis AP, Altaweel M,Aiello LP, Bressler NM, D'Amico DJ, Goldbaum M, Guyer DR, Katz B, Patel M, Schwartz SD; Macugen Diabetic Retinopathy Study Group. A phase II randomized double-masked trial of pegaptanib, an anti-vascular endothelial growth factor aptamer, for diabetic macular edema. Ophthalmology. 2005; 112(10):1747-57 
14. Murphy RP, Nanda M, Plotnick L, Enger C, Vitale S, Patz A The relationship of puberty to diabetic retinopathy. Arch Ophthalmol. 1990; 108(2): 215-8.

15. Merimee TJ, Zapf J, Froesch ER. Insulin-like growth factors. Studies in diabetics with and without retinopathy. N Engl J Med. 1983; 309(9): 527-30.

16. Grant M, Russell B, Fitzgerald C, Merimee TJ. Insulin-like growth factors in vitreous. Studies in control and diabetic subjects with neovascularization. Diabetes. 1986; 35(4): 416-20.

17. Chanteleau E, Kohner EM. Why some cases of retinopathy worsen when diabetic control improves. BMJ. 1997; 315(7116): 1105-6

18. Smith LE, Kopchick JJ, Chen W, Knapp J, Kinose F, Daley $D$, et al. Essential role of growth hormone in ischemiainduced retinal neovascularization. Science. 1997; 276(5319): 1706-9.

19. Kuwabara T, Cogan DG. Retinal vascular patterns. VI. Mural cells of the retinal capillaries. Arch Ophthalmol. 1963; 69: 492-502.
20. Antcliff RJ, Marshall J. The pathogenesis of edema in diabetic maculopathy. Semin Ophthalmol. 1999; 14(4): 223-32.

21. Basteau F, Montermousque B, Aouizerate F. CEdème maculaire diffus de la rétinopathie diabétique et pathologie de l'interface vitréo-rétinienne. J Fr Ophtalmol. 2004 ; 27(10): 1109-20.

\section{AUTOR CORRESPONDENTE:}

Jacqueline Coblentz

Rua Visconde de Silva, 52/703

22271-090 - Rio de Janeiro - RJ

Tel/fax: 2286-5744 ou 2266-3710

E-mail: j_coblentz@yahoo.com 